\title{
Fundamentos de la Olimpiada Paraguaya de Astronomía y Astronáutica
}

\author{
Eduardo Quintana \\ Encargaduría Cultura Científica \\ Universidad Iberoamericana \\ eduardoquintanaelsebf@gmail.com
}

\section{INTRODUCCIÓN}

La astronomía paraguaya todavía es una materia pendiente en la academia. No existen ni centros ni grupos de investigación en la materia, salvo casos excepcionales e individuales. En la actualidad existen cuatro asociaciones grandes de astronomía a nivel nacional, pero no existen carreras universitarias relacionadas a la astronomía ni a la astrofísica. Es por ello que los principales núcleos de divulgación y abordaje se desarrollan mejor en las instituciones educativas de nivel básico y medio y en los grupos de divulgación. Es por ello emblemático el trabajo realizado por el Club de Astrofísica del Paraguay con la Olimpiada Paraguaya de Astronomía y Astronáutica (OPAA), un espacio que permite acercar la ciencia a los estudiantes y despertar las vocaciones científicas de manera temprana.

\section{Decano de La AStronomía local}

Durante la era colonial, la Provincia del Paraguay albergaba investigaciones en astronomía. En la compañía jesuítica de San Cosme y Damián, el padre Buenaventura Suárez desarrolló en el siglo XVIII trabajos científicos de astronomía, en medio de la precaridad de la zona y con instrumentos construidos por él mismo. Pudo escribir Lunario de un siglo, un libro científico y divulgativo de gran relevancia para la ciencia de la época. ${ }^{1}$ Pero la historia de la astronomía no pudo desarrollarse cabalmente, ya que las instituciones académicas y científicas locales no se interesaron en su progreso ni avance. A pesar de ello, se crearon clubes y grupos astronómicos independientes, constituyéndose como fundaciones o organizaciones no gubernamentales.

"A finales de 1985, un grupo de ingenieros y profesionales de diversas ramas se organizaron para preparar un evento sensacional: el paso del Halley, que se da cada 76 años. Motivados por la presencia en Paraguay

'Suárez,íconodelacienciasudamericana(2014).DiarioABCColor-SuplementoCultural.Recuperadode: http://www.abc.com.py/edicion-impresa/suplementos/cultural/buenaventura-suarez-icono-de-la-cienciasudamericana-1210726.html. 


\section{Proyectos de la UNIBE}

de los astrónomos Richard West y Jorge Sahade, de la Unión Astronómica Internacional, se organizó un debate en la Facultad de Ingeniería de la UNA, que duró desde las 19:00 hasta las 02:00 am del día siguiente. Luego de eso comenzaron las reuniones sabatinas $y$, aunque tenían escasos telescopios y binoculares, con una gran pasión por los astros fundaron el Club de Astrofísica del Paraguay, que llegó a tener 53 socios en los primeros tiempos. Un poco más tarde realizaron en total 200 charlas entre 1985 y 1986, en diversas instituciones educativas, culturales y deportivas para que nadie se pierda del Halley."2

En 1985 se funda el Club de Astrofísica del Paraguay (CAP). A inicios de la decada del noventa, se crea la Asociación de Aficionados a la Astronomía que hoy se transformó en el Centro Astronómico Bicentenario. También en los noventas se formó la Sociedad de Estudios Astronómicos (SEA) y en el año 2009, se creó el Centro de Difusión de Investigación Astronómica (CEDIA). A nivel institucional, el Planetario Buenaventura Suárez hoy depende de la Municipalidad de Asunción y el Centro Astronómico de San Cosme y Damián dependen de la Municipalidad de San Cosme y de la Secretaría Nacional de Turismo. También sigue activo el Centro Paraguayo de Información Astronómica (CPIA).

Es el decano de la astronomía paraguaya el que lleva adelante la Olimpiada Paraguaya de Astronomía y Astronáutica (OPAA). En 2009 se celebró el Año Internacional de la Astronomía, que tuvo al CAP como organizador y nodo principal del evento en nuestro país. La inauguración se llevó a cabo en la ciudad de Concepción. Sin embargo, uno de los proyectos fue la creación de la OPAA.

Según el CAP, la OPAA es un evento de competición académica a nivel nacional que permite acercar la ciencia a los estudiantes.

"La Olimpiada Paraguaya de Astrofísica y Astronáutica (OPAA) es un evento académico de competencia y confraternidad estudiantil entre estudiantes del Sistema de Educativo Paraguayo, pertenecientes a la Educación Escolar Básica y Media, de todo el territorio nacional, inscriptos en Instituciones Educativas Públicas y Privadas."

\section{Apoyo de LA UnIBE}

El evento se lleva a cabo cada año en los diferentes departamentos del país, en las instituciones que colaboran con el CAP. Hasta el año 2015, la Organización Multidisciplinaria de Apoyo a Profesores y Alumnos (Omapa), fue la institución cooperadora del evento. Sin embargo, a partir del 2016, el apoyo a la institución astronómica lo da la Universidad Iberoamericana.

\footnotetext{
2EldecanodelaastronomíaenelParaguaycumple30añosdevida(2015).DiarioABCColor-Suplemento deCienciayTecnología.Recuperadode:http://www.abc.com.py/edicion-impresa/ciencia-y-tecnologia/eldecano-de-la-astronomia-en-el-paraguay-cumple-30-anos-de-vida-1435791.html.

${ }^{3}$ Proyecto de la OPAA (2016). Club de Astrofísica del Paraguay. 
"La Unibe asume el compromiso de llevar adelante esta Olimpiada, de suma importancia para el desarrollo de las capacidades científicas y tecnológicas, con el fin de promover el desarrollo de capacidades científicas y tecnológicas e identificar talentos científicos en estudiantes de la Educación Media y Educación Escolar Básica del Paraguay, como aporte al desarrollo científico, productivo, económico y social del país"."

Entonces, el objetivo principal de la OPAA es fomentar el entusiasmo estudiantil por la ciencia y la tecnología y a la vez identificar los jóvenes talentos de la educación paraguaya, en las áreas de astronomía y astronáutica.

En en plan del 2016, el Club de Astrofísica también incluía los objetivos más específicos:

a) Incentivar la participación de los diferentes grupos nacionales de Astronomía.

b) Elevar el nivel de conocimientos sobre la Astronomía, Astronáutica y Ciencias afines.

c) Seleccionar a los representantes del país para la Olimpiada Lationamericana de Astronomía y Astronáutica (OLAA).

d)Participar en la Olimpiada Latinoamericana de Astronomía y Astronáutica (OLAA), a desarrollarse en la Ciudad de Córdoba - Argentina.

EI CAP desarrolló clases teóricas y prácticas con los estudiantes sobre ensayo planetario, manejo de telescopio, observaciones astronómicas, construcción y lanzamiento de cohetes hidroneumáticos. Para la clasificación, se tomaron varios exámenes y los mejores representaron al Paraguay en la Olimpiada Latinoamericana de Astronomía y Astronáutica, realizada en la ciudad de Córdoba. El evento tuvo su cierre en el mes de octubre de 2016 en la Unibe. En total, participaron 110 estudiantes del nivel Escolar Básico y nivel Medio de todo el país.

\section{Olimpiada COMO EDUCACIÓN ASTRONÓMICA}

El trabajo del CAP se encuadra dentro de la educación no formal de la ciencia. La astronomía se constituye como un elemento alternativo al programa de planes de estudio vigente por el Ministerio de Educación y Cultura. La filosofía subyacente a la Olimpiada Paraguaya de Astronomía y Astronáutica es el acercamiento indirecto de una ciencia para despertar el interés y el descubrimiento de jóvenes talentos científicos.

En 2016 se realizó la octava edición de la OPAA, evidenciando un intento de institucionalización del evento. La iniciativa está liderada, desde sus inicios, por el ingeniero Miguel Ángel Volpe, catedrático de la Universidad Nacional de Asunción y presidente del Club de Astrofísica del Paraguay. Volpe, uno de los principales divulgadores científicos de Paraguay, quien fue homenajeado en 2016 por la Unión Astronómica Internacional (UAI) con el nombramiento de un asteroide en su honor.

${ }^{4}$ Íbid 


\section{Proyectos de la UNIBE}

"El asteroide 16701 Volpe es un cuerpo del cinturón principal descubierto el 21 de febrero de 1995 en el observatorio Kitt Peak por el Programa Spachewatch. La nominación fue propuesta por Willem Albertus Froguer. Su periodo de revolución alrededor del Sol es de 1356 días (3.71 años). Posee una magnitud absoluta de 14,4 y su tamaño se estiman entre $3,5 \mathrm{~km}$ y 7,5 km", informó el Centro Paraguayo de Informaciones Astronómicas. ${ }^{5}$

El trabajo de Volpe constituye un aporte más a la educación de la astronomía, que podría acompañarse desde otras instituciones, para incentivar una mayor participación estudiantil y mejor infraestructura del evento en sí.

Los objetivos que persigue la OPAA podrían canalizarse a través de políticas científicas más abarcantes, que deberían incluir estudios sobre la posibilidad de apertura de carreras universitarias relacionadas a las ciencias astronómicas y espaciales. Ya que de esta manera no se cortaría el proyecto original del CAP y se daría continuidad a la vocación científica en el área de la astronomía y la astronáutica.

\section{BibLIOGRAFÍA}

\section{Documentos}

Proyecto de la Olimpiada Paraguaya de Astronomía y Astronáutica (2016). Club de Astrofísica del Paraguay

\section{Internet}

El decano de la astronomía en el Paraguay cumple 30 años de vida (2015). Diario ABC Color, Suplemento de Ciencia y Tecnología. Recuperado de: http:// www.abc.com.py/edicion-impresa/ciencia-y-tecnologia/el-decano-de-laastronomia-en-el-paraguay-cumple-30-anos-de-vida-1435791.html.

Paraguay tiene un nuevo asteroide: 16701 Volpe (2016). Diario ABC Color. Recuperado de: http://www.abc.com.py/edicion-impresa/locales/paraguaytiene-un-nuevo-asteroide-16701-volpe-1493777.html.

Suárez, ícono de la ciencia sudamericana (2014). Diario ABC Color Suplemento Cultural. Recuperado de: http://www.abc.com.py/edicionimpresa/suplementos/cultural/buenaventura-suarez-icono-de-la-cienciasudamericana-1210726.html.

${ }^{5}$ Paraguaytieneunnuevoasteroide:16701Volpe(2016).DiarioABCColor.Recuperadode:http://www.abc. com.py/edicion-impresa/locales/paraguay-tiene-un-nuevo-asteroide-16701-volpe-1493777.html 